\title{
Compressions of $k$ th-order slant Toeplitz operators to model spaces
}

\author{
Bartosz Lanucha and Małgorzata Michalska \\ Institute of Mathematics, Maria Curie-Skłodowska University, pl. M. Curie-Skłodowskiej 1, 20-031 Lublin, Poland \\ (e-mail: bartosz.lanucha@poczta.umcs.lublin.pl; malgorzata.michalska@poczta.umcs.lublin.pl)
}

Received November 23, 2020; revised May 21, 2021

\begin{abstract}
In this paper, we consider compressions of $k$ th-order slant Toeplitz operators to the backward shift-invariant subspaces of the classical Hardy space $H^{2}$. In particular, we characterize these operators using compressed shifts and finite-rank operators of special kind.
\end{abstract}

MSC: 47B32, 47B35, 30H10

Keywords: model space, compressed shift, Toeplitz operator, slant Toeplitz operator, generalized slant Toeplitz operator, truncated Toeplitz operator

\section{Introduction}

Let $\mathbb{T}=\{z \in \mathbb{C}:|z|=1\}$, and denote by $L^{2}=L^{2}(\mathbb{T})$ the space of Lebesgue-measurable square-integrable functions on $\mathbb{T}$ and by $L^{\infty}=L^{\infty}(\mathbb{T})$ the space of Lebesgue-measurable essentially bounded functions. Moreover, let $H^{2}$ be the classical Hardy space in the disk $\mathbb{D}=\{z \in \mathbb{C}:|z|<1\}$. As usual, we view $H^{2}$ as a space of functions analytic in $\mathbb{D}$ or (via radial limits) as a closed subspace of $L^{2}$ (see $[11,13]$ for details). We denote by $P$ the orthogonal projection from $L^{2}$ onto $H^{2}$.

For $\varphi \in L^{\infty}$, the classical Toeplitz operator $T_{\varphi}$ is defined on $H^{2}$ by

$$
T_{\varphi} f=P M_{\varphi} f, \quad f \in H^{2},
$$

where $M_{\varphi}: L^{2} \rightarrow L^{2}$ is the multiplication operator $f \mapsto \varphi f$. For $\varphi \in L^{2}$, the operators $T_{\varphi}$ and $M_{\varphi}$ can be defined on a dense subset of $H^{2}$ and $L^{2}$, respectively (namely, on bounded functions).

Classical Toeplitz operators, as compressions of multiplication operators to $H^{2}$, are among the most studied linear operators on the Hardy space. Their study goes back to the beginning of the 20th century (for details and references, see, e.g., $[3,28])$. In recent years, compressions of multiplication operators to model spaces were widely studied. Model spaces are the nontrivial subspaces of $H^{2}$ that are invariant for the backward shift operator $S^{*}=T_{\bar{z}}$. Each of these subspaces is of the form $K_{\alpha}=H^{2} \ominus \alpha H^{2}$, where $\alpha$ is a nonconstant inner function, a function analytic and bounded in $\mathbb{D}\left(\alpha \in H^{\infty}\right)$ such that its boundary values are of modulus one a.e. on $\mathbb{T}$ (if $\alpha$ is constant, then $K_{\alpha}=\{0\}$ ). 
Each model space $K_{\alpha}$ is a reproducing kernel Hilbert space with the reproducing kernel function $k_{w}^{\alpha}$ at a point $w \in \mathbb{D}$ given by

$$
k_{w}^{\alpha}(z)=\frac{1-\overline{\alpha(w)} \alpha(z)}{1-\bar{w} z}, \quad z \in \mathbb{D} .
$$

Note that for each $w \in \mathbb{D}$, we have $k_{w}^{\alpha} \in K_{\alpha}^{\infty}=K_{\alpha} \cap H^{\infty}$. As the linear span of $\left\{k_{w}^{\alpha}: w \in \mathbb{D}\right\}$ is dense in $K_{\alpha}$, we see that $K_{\alpha}^{\infty}$ is a dense subset of $K_{\alpha}$.

The orthogonal projection $P_{\alpha}$ from $L^{2}$ onto $K_{\alpha}$ is given by $P_{\alpha}=P-M_{\alpha} P M_{\bar{\alpha}}$ (see [12, Cor. 14.13]). In particular, $P_{\alpha \mid H^{2}}=I_{H^{2}}-T_{\alpha} T_{\bar{\alpha}}$.

It is easy to see that if $\alpha(z)=z^{n}$, then $K_{\alpha}$ is the set of all polynomials of degree at most $n-1$. In that case, $\operatorname{dim} K_{\alpha}=n$, and $\left\{1, z, \ldots, z^{n-1}\right\}$ is an orthonormal basis for $K_{\alpha}$. It is known (see [13]) that $\operatorname{dim} K_{\alpha}<\infty$ if and only if $\alpha$ is a finite Blaschke product. For example, if $\alpha$ is a finite Blaschke product with $n$ distinct zeros $a_{1}, \ldots, a_{n}$, then the set of corresponding kernel functions $k_{a_{1}}^{\alpha}, \ldots, k_{a_{n}}^{\alpha}$ is a (nonorthogonal) basis for $K_{\alpha}$ (see $[12,13])$.

Model spaces are important in view of their connection with various topics, one being the Sz.-Nagy-Foias model theory [30, Chap. 13]. For more details on properties and structure of model spaces, see [13] and [12].

For an inner function $\alpha$ and for $\varphi \in L^{2}$, let

$$
A_{\varphi}^{\alpha} f=P_{\alpha}(\varphi f), \quad f \in K_{\alpha}^{\infty},
$$

where $P_{\alpha}$ is the orthogonal projection from $L^{2}$ onto $K_{\alpha}$. Note that $A_{\varphi}^{\alpha}$ is densely defined since $K_{\alpha}^{\infty}$ is a dense subset of $K_{\alpha}$. These operators, called truncated Toeplitz operators, gained attention in 2007 with Sarason's paper [29]. Ever since, truncated Toeplitz operators have been under constant intensive study, which led to many interesting results and applications (see $[8,15]$ and references therein). More recently, the authors of $[6,7]$ and [4] introduced the so-called asymmetric truncated Toeplitz operators: for two inner functions $\alpha, \beta$ and for $\varphi \in L^{2}$, the asymmetric truncated Toeplitz operator $A_{\varphi}^{\alpha, \beta}$ is defined by

$$
A_{\varphi}^{\alpha, \beta} f=P_{\beta}(\varphi f), \quad f \in K_{\alpha}^{\infty} .
$$

As above, $A_{\varphi}^{\alpha, \beta}$ is densely defined. Moreover, $A_{\varphi}^{\alpha, \alpha}=A_{\varphi}^{\alpha}$. These operators were then studied in, for example, $[5,17,22,23,24,25]$.

Recall that if $\varphi(z)=\sum_{j=-\infty}^{\infty} a_{j} z^{j} \in L^{\infty}$, then for each $n \in \mathbb{Z}, M_{\varphi}\left(z^{n}\right)=\sum_{j=-\infty}^{\infty} a_{j-n} z^{j}$, and the matrix of $M_{\varphi}$ with respect to the standard basis $\left\{z^{n}: n \in \mathbb{Z}\right\}$ is given by

$$
\left[\begin{array}{cccccc}
\ddots & \vdots & \vdots & \vdots & \vdots & \\
\ldots & a_{0} & a_{-1} & a_{-2} & a_{-3} & \ldots \\
\ldots & a_{1} & a_{0} & a_{-1} & a_{-2} & \ldots \\
\ldots & a_{2} & a_{1} & a_{0} & a_{-1} & \ldots \\
\ldots & a_{3} & a_{2} & a_{1} & a_{0} & \ldots \\
& \vdots & \vdots & \vdots & \vdots & \ddots
\end{array}\right]
$$

Similarly, for each $n \in \mathbb{N}_{0}, T_{\varphi}\left(z^{n}\right)=\sum_{j=0}^{\infty} a_{j-n} z^{j}$, and $T_{\varphi}$ is the operator on $H^{2}$ represented by the matrix (with respect to $\left\{z^{n}: n \in \mathbb{N}_{0}\right\}$ )

$$
\left[\begin{array}{ccccc}
a_{0} & a_{-1} & a_{-2} & a_{-3} & \ldots \\
a_{1} & a_{0} & a_{-1} & a_{-2} & \ldots \\
a_{2} & a_{1} & a_{0} & a_{-1} & \ldots \\
a_{3} & a_{2} & a_{1} & a_{0} & \ldots \\
\vdots & \vdots & \vdots & \vdots & \ddots
\end{array}\right]
$$


In [1,2], for $k \in \mathbb{N}, k \geqslant 2$, and $\varphi(z)=\sum_{j=-\infty}^{\infty} a_{j} z^{j} \in L^{\infty}$, the authors define the $k$ th-order slant Toeplitz operator $U_{\varphi}$ on $L^{2}$ by $U_{\varphi}\left(z^{n}\right)=\sum_{j=-\infty}^{\infty} a_{k j-n} z^{j}$ for $n \in \mathbb{Z}$, that is, as the operator on $L^{2}$ represented by the matrix

$$
\left[\begin{array}{cccccc}
\ddots & \vdots & \vdots & \vdots & \vdots & \\
\ldots & a_{0} & a_{-1} & a_{-2} & a_{-3} & \ldots \\
\ldots & a_{k} & a_{k-1} & a_{k-2} & a_{k-3} & \ldots \\
\ldots & a_{2 k} & a_{2 k-1} & a_{2 k-2} & a_{2 k-3} & \ldots \\
\ldots & a_{3 k} & a_{3 k-1} & a_{3 k-2} & a_{3 k-3} & \ldots \\
& \vdots & \vdots & \vdots & \vdots & \ddots
\end{array}\right] .
$$

Equivalently, $U_{\varphi}: L^{2} \rightarrow L^{2}, \varphi \in L^{\infty}$, is given by

$$
U_{\varphi} f=W_{k} M_{\varphi} f, \quad f \in L^{2}, \quad \text { where } \quad W_{k}\left(z^{n}\right)= \begin{cases}z^{m} & \text { if } \frac{n}{k}=m \in \mathbb{Z}, \\ 0 & \text { if } \frac{n}{k} \notin \mathbb{Z} .\end{cases}
$$

The authors of $[1,2]$ also consider $V_{\varphi}$, the compression of $U_{\varphi}$ to $H^{2}$, defined by

$$
V_{\varphi} f=P U_{\varphi} f, \quad f \in H^{2}
$$

and represented by the matrix

$$
\left[\begin{array}{ccccc}
a_{0} & a_{-1} & a_{-2} & a_{-3} & \ldots \\
a_{k} & a_{k-1} & a_{k-2} & a_{k-3} & \ldots \\
a_{2 k} & a_{2 k-1} & a_{2 k-2} & a_{2 k-3} & \ldots \\
a_{3 k} & a_{3 k-1} & a_{3 k-2} & a_{3 k-3} & \ldots \\
\vdots & \vdots & \vdots & \vdots & \ddots
\end{array}\right]
$$

Note that if $\varphi \in L^{2}$, then $U_{\varphi}$ and $V_{\varphi}$ are densely defined. For $k=2$, the operators $U_{\varphi}$ (called slant Toeplitz operators) and their compressions to $H^{2}$ were first studied in $[18,32]$ (see also $[19,20,21]$ ). These operators have connections with wavelet theory and dynamical systems (see, e.g., $[16,20,31]$ ). In the more recent papers $[9,10]$, slant Toeplitz operators in multivariable setting are considered. In [26, 27] the authors investigate the commutativity of $k$ th-order slant Toeplitz operators. Observe that if $k=1$ in the above definitions, then $U_{\varphi}=M_{\varphi}$ and $V_{\varphi}=T_{\varphi}$.

Here we study compressions of $k$ th-order slant Toeplitz operators to model spaces. For a fixed $k \in \mathbb{N}$, two inner functions $\alpha, \beta$, and for $\varphi \in L^{2}$, define

$$
U_{\varphi}^{\alpha, \beta} f=P_{\beta} U_{\varphi} f=P_{\beta} W_{k}(\varphi f), \quad f \in K_{\alpha}^{\infty},
$$

and denote by $\mathcal{S}_{k}(\alpha, \beta)$ the set of all the compressions $U_{\varphi}^{\alpha, \beta}, \varphi \in L^{2}$, that can be boundedly extended to $K_{\alpha}$.

In Section 2, we investigate conditions on $\varphi$ that imply that $U_{\varphi}^{\alpha, \beta}=0$. We show that, in contrast with the case of $U_{\varphi}$ and $V_{\varphi}$ [1,2], $U_{\varphi}^{\alpha, \beta}$ can be equal to the zero operator for $\varphi$ not equal to zero.

In Section 3, we characterize operators from $\mathcal{S}_{k}(\alpha, \beta)$ using compressed shifts $S_{\alpha}=A_{z}^{\alpha}$ and $S_{\beta}=A_{z}^{\beta}$. It is well known that a bounded linear operator $T: H^{2} \rightarrow H^{2}$ is a Toeplitz operator if and only if $T-S^{*} T S=0$, where $S$ is the shift operator $S=T_{z}$. A similar characterization was given in [1,2] for $V_{\varphi}$. Namely, $T$ is a compression of a $k$ th-order slant Toeplitz operator to $H^{2}$ if and only if $T-S^{*} T S^{k}=0$. We show, for example, that a bounded linear operator $U$ from $K_{\alpha}$ into $K_{\beta}$ belongs to $\mathcal{S}_{k}(\alpha, \beta)$ if and only if $U-S_{\beta} U\left(S_{\alpha}^{*}\right)^{k}$ is a special kind of operator of rank at most $k+1$. This is done in the spirit of Sarason's characterization of truncated Toeplitz operators given in [29], where, among other results, he shows that $U$ is a truncated Toeplitz 
operator if and only if $U-S_{\alpha} U S_{\alpha}^{*}$ is of rank two and special kind (see also $[4,17,24]$ for the asymmetric case).

In Section 4, we use the results obtained to show that for $k \leqslant \operatorname{dim} K_{\alpha}$, the operator $U_{\varphi}^{\alpha, \beta}$ is zero if and only if $\varphi$ belongs to $\overline{\alpha H^{2}}+\bar{z}^{k-1}\left(W_{k}^{*} \beta\right) H^{2}$.

\section{Operators from $\mathcal{S}_{k}(\alpha, \beta)$ equal to the zero operator, part 1}

In this section, we investigate for which $\varphi \in L^{2}, U_{\varphi}^{\alpha, \beta}=0$.

We start with some basic properties of the operator $W_{k}$ and its adjoint $W_{k}^{*}$. Some of these properties can be found, for example, in [18] (for $k=2$ ) and [1,2].

Lemma 1. Let $k \in \mathbb{N}$. Then:

(a) $W_{k}^{*} f(z)=f\left(z^{k}\right),|z|=1, f \in L^{2}$;

(b) $W_{k}^{*}(f \cdot g)=W_{k}^{*} f \cdot W_{k}^{*} g$ for all $f, g \in L^{2}$ such that $f \cdot g \in L^{2}$;

(c) $W_{k} W_{k}^{*}=I_{L^{2}}$, and $W_{k}^{*} W_{k}$ is the orthogonal projection from $L^{2}$ onto the closed linear span of $\left\{z^{k m}: m \in \mathbb{Z}\right\}$;

(d) $W_{k} \bar{f}=\overline{W_{k} f}$ and $W_{k}^{*} \bar{f}=\overline{W_{k}^{*} f}, f \in L^{2}$;

(e) $P$ reduces $W_{k}$, that is, $P W_{k}=W_{k} P$ and $P W_{k}^{*}=W_{k}^{*} P$;

(f) $M_{\varphi} W_{k}=W_{k} M_{W_{k}^{*} \varphi}$ for every $\varphi \in L^{\infty}$;

(g) for every inner function $\alpha$, the function $W_{k}^{*} \alpha$ is also inner, and $P_{\alpha} W_{k}=W_{k} P_{W_{k}^{*} \alpha}$.

Proof. The proofs of (a)-(e) are straightforward. We only prove (f) and (g).

To show (f), fix $\varphi \in L^{\infty}$ and take any $f, g \in L^{2}$. Then by (b) and (d)

$$
\left\langle M_{\varphi} W_{k} f, g\right\rangle=\left\langle f, W_{k}^{*}(\bar{\varphi} g)\right\rangle=\left\langle f, \overline{W_{k}^{*} \varphi} \cdot W_{k}^{*} g\right\rangle=\left\langle W_{k} M_{W_{k}^{*} \varphi} f, g\right\rangle .
$$

If $\alpha$ is an inner function, then $W_{k}^{*} \alpha$ is also an inner function by (a). Since $P_{\alpha}=P-M_{\alpha} P M_{\bar{\alpha}}$, using (e) and (f), we obtain

$$
\begin{aligned}
P_{\alpha} W_{k} & =P W_{k}-M_{\alpha} P W_{k} M_{\overline{W_{k}^{*} \alpha}}=W_{k} P-M_{\alpha} W_{k} P M_{\overline{W_{k}^{*} \alpha}} \\
& =W_{k}\left(P-M_{W_{k}^{*} \alpha} P M_{\overline{W_{k}^{*} \alpha}}\right)=W_{k} P_{W_{k}^{*} \alpha},
\end{aligned}
$$

that is, (g) holds.

Note that by (e), (f), and (g) we get that for $\varphi \in L^{\infty}$,

$$
T_{\varphi} W_{k}=W_{k} T_{W_{k}^{*} \varphi} \quad \text { and } \quad W_{k}^{*} T_{\varphi}=T_{W_{k}^{*} \varphi} W_{k}^{*} \quad \text { on } H^{2} .
$$

For the remainder of this section, fix $k \in \mathbb{N}$.

Proposition 1. Let $\alpha$ and $\beta$ be two inner functions, and let $\varphi \in L^{2}$. If $\varphi \in \overline{\alpha H^{2}}+\left(W_{k}^{*} \beta\right) H^{2}$, then $U_{\varphi}^{\alpha, \beta}=0$.

Proof. Assume that $\varphi=\overline{\alpha h_{1}}+W_{k}^{*} \beta \cdot h_{2}$ for some $h_{1}, h_{2} \in H^{2}$. Then for all $f \in K_{\alpha}^{\infty}$ and $g \in K_{\beta}^{\infty}$, we have (by Lemma $1(\mathrm{~g})$ )

$$
\begin{aligned}
\left\langle U_{\varphi}^{\alpha, \beta} f, g\right\rangle & =\left\langle\varphi f, W_{k}^{*} g\right\rangle=\left\langle\overline{\alpha h_{1}} f, W_{k}^{*} g\right\rangle+\left\langle W_{k}^{*} \beta \cdot h_{2} f, W_{k}^{*} g\right\rangle \\
& =\left\langle f, \alpha h_{1} \cdot W_{k}^{*} g\right\rangle+\left\langle P_{\beta} W_{k}\left(W_{k}^{*} \beta \cdot h_{2} f\right), g\right\rangle \\
& =\left\langle W_{k} P_{W_{k}^{*} \beta}\left(W_{k}^{*} \beta \cdot h_{2} f\right), g\right\rangle=0 .
\end{aligned}
$$


Corollary 1. If $U \in \mathcal{S}_{k}(\alpha, \beta)$, then $U=U_{\varphi}^{\alpha, \beta}$ with $\varphi$ from $\overline{K_{\alpha}}+K_{W_{k}^{*} \beta}$.

Hence $U_{\varphi}^{\alpha, \beta}$ is not uniquely determined by its symbol. Moreover, the boundedness of a symbol is not necessary for the boundedness of $U_{\varphi}^{\alpha, \beta}$.

In general, the implication in Proposition 1 cannot be reversed as the following example shows.

Example 1. Let $k=2, \alpha(z)=z^{4}$, and $\beta(z)=z^{3}$. In that case, for $\varphi=\sum_{n=-\infty}^{\infty} a_{n} z^{n} \in L^{2}$, the operator $U_{\varphi}^{\alpha, \beta}$ is represented by the matrix

$$
\left[\begin{array}{cccc}
a_{0} & a_{-1} & a_{-2} & a_{-3} \\
a_{2} & a_{1} & a_{0} & a_{-1} \\
a_{4} & a_{3} & a_{2} & a_{1}
\end{array}\right] .
$$

Hence if, for example, $\varphi(z)=z^{5}$, then $U_{\varphi}^{\alpha, \beta}=0$. But here $z^{5} \notin \overline{\alpha H^{2}}+\left(W_{k}^{*} \beta\right) H^{2}=\overline{z^{4} H^{2}}+z^{6} H^{2}$.

Observe that $U_{\varphi}^{\alpha, \beta}$ can be seen as a composition of $W_{k}$ and an asymmetric truncated Toeplitz operator. Indeed, using Lemma $1(\mathrm{~g})$, for $f \in K_{\alpha}^{\infty}$, we get

$$
U_{\varphi}^{\alpha, \beta} f=P_{\beta} W_{k}(\varphi f)=W_{k} P_{W_{k}^{*} \beta}(\varphi f)=W_{k} A_{\varphi}^{\alpha, W_{k}^{*} \beta} f .
$$

Now Proposition 1 follows from the fact that $A_{\varphi}^{\alpha, \beta}=0$ if and only if $\varphi \in \overline{\alpha H^{2}}+\beta H^{2}$ (see [4,22]).

Let $\alpha$ be an inner function. Then the operator $C_{\alpha}: L^{2} \rightarrow L^{2}$ defined by

$$
C_{\alpha} f(z)=\alpha(z) \bar{z} \overline{f(z)}, \quad|z|=1,
$$

is a conjugation on $L^{2}$ (an antilinear isometric involution). Moreover, $C_{\alpha}\left(K_{\alpha}\right)=K_{\alpha}$ (see [14] and [13, Chap. 8]). It is known that all truncated Toeplitz operators are $C_{\alpha}$-symmetric, that is, $C_{\alpha} A_{\varphi}^{\alpha} C_{\alpha}=\left(A_{\varphi}^{\alpha}\right)^{*}=A_{\bar{\varphi}}^{\alpha}$ [29]. It was observed in [24] that $C_{\beta} A_{\varphi}^{\alpha, \beta} C_{\alpha}=A_{\frac{\alpha \varphi \beta}{\alpha, \beta}}$ (see also [17]). Here we have the following:

Proposition 2. Let $\alpha, \beta$ be two inner functions, and let $\varphi \in L^{2}$. Then (on $K_{\alpha}^{\infty}$ ) $C_{\beta} U_{\varphi}^{\alpha, \beta} C_{\alpha}=U_{\psi}^{\alpha, \beta}$ with $\psi=\overline{z^{k-1} \alpha \varphi} W_{k}^{*} \beta$.

Proof. Let $\varphi \in L^{2}$. Then for $f \in K_{\alpha}^{\infty}$ and $g \in K_{\beta}^{\infty}$, by Lemma 1 we have

$$
\begin{aligned}
\left\langle C_{\beta} U_{\varphi}^{\alpha, \beta} C_{\alpha} f, g\right\rangle & =\left\langle C_{\beta} P_{\beta} W_{k} M_{\varphi} C_{\alpha} f, g\right\rangle=\left\langle C_{\beta} g, P_{\beta} W_{k}\left(\varphi C_{\alpha} f\right)\right\rangle \\
& =\left\langle C_{\beta} g, W_{k}\left(\varphi C_{\alpha} f\right)\right\rangle=\left\langle\bar{z} \beta \bar{g}, W_{k}(\varphi \bar{z} \alpha \bar{f})\right\rangle \\
& =\left\langle W_{k}^{*}(\bar{z} \beta \bar{g}), \varphi \bar{z} \alpha \bar{f}\right\rangle=\left\langle\overline{z^{k-1} \alpha \varphi} W_{k}^{*} \beta \cdot f, W_{k}^{*} g\right\rangle \\
& =\left\langle P_{\beta} W_{k} M_{\overline{z^{k-1} \alpha \varphi} W_{k}^{*} \beta} f, g\right\rangle=\left\langle U_{\overline{z^{k-1} \alpha \varphi} W_{k}^{*} \beta} f, g\right\rangle .
\end{aligned}
$$

As a corollary of Proposition 2, we get the following:

Corollary 2. Let $\alpha, \beta$ be two inner functions, and let $U$ be a bounded linear operator from $K_{\alpha}$ into $K_{\beta}$. Then $U \in \mathcal{S}_{k}(\alpha, \beta)$ if and only if $C_{\beta} U C_{\alpha} \in \mathcal{S}_{k}(\alpha, \beta)$.

Proposition 3. Let $\alpha, \beta$ be two inner functions, and let $\varphi \in L^{2}$. If $\varphi \in \overline{\alpha H^{2}}+\bar{z}^{k-1}\left(W_{k}^{*} \beta\right) H^{2}$, then $U_{\varphi}^{\alpha, \beta}=0$. 
Proof. Let $\varphi=\overline{\alpha h_{1}}+\bar{z}^{k-1} W_{k}^{*} \beta \cdot h_{2}$ with $h_{1}, h_{2} \in H^{2}$. By Proposition 1 we have $U_{\bar{\alpha}, \beta}^{\alpha, \beta}=0$ and, consequently, $U_{\varphi}^{\alpha, \beta}=U_{\bar{z}^{k-1} W_{k}^{*} \beta \cdot h_{2}}^{\alpha}$. Now, using Proposition 2, we obtain

$$
C_{\beta} U_{\varphi}^{\alpha, \beta} C_{\alpha}=C_{\beta} U_{\bar{z}^{k-1} W_{k}^{*} \beta \cdot h_{2}}^{\alpha, \beta} C_{\alpha}=U_{\frac{z^{k-1} \alpha \bar{z}^{k-1} W_{k}^{*} \beta \cdot h_{2}}{\alpha, \beta} W_{k}^{*} \beta}=U_{\overline{\alpha h_{2}}}^{\alpha, \beta}=0,
$$

from which it follows that $U_{\varphi}^{\alpha, \beta}=0$.

Corollary 3. If $U \in \mathcal{S}_{k}(\alpha, \beta)$, then $U=U_{\varphi}^{\alpha, \beta}$ with $\varphi$ from $\overline{K_{\alpha}}+\bar{z}^{k-1} K_{W_{k}^{*} \beta}$.

Note that

$$
\overline{\alpha H^{2}}+\left(W_{k}^{*} \beta\right) H^{2}=\overline{\alpha H^{2}}+\bar{z}^{k-1}\left(W_{k}^{*} \beta\right) z^{k-1} H^{2} \subset \overline{\alpha H^{2}}+\bar{z}^{k-1}\left(W_{k}^{*} \beta\right) H^{2} .
$$

Moreover, if an inner function $\beta$ is such that $\beta(0)=0$, then $z^{k}$ divides $W_{k}^{*} \beta(z)=\beta\left(z^{k}\right)$, and $\bar{z}^{k-1} W_{k}^{*} \beta$ is also an inner function, $\bar{z}^{k-1}\left(W_{k}^{*} \beta\right) H^{2} \subset z H^{2}$.

\section{Characterizations of operators from $\mathcal{S}_{k}(\alpha, \beta)$}

Let $\alpha$ and $\beta$ be two nonconstant inner functions. In this section, we characterize operators from $\mathcal{S}_{k}(\alpha, \beta)$, $k \in \mathbb{N}$, using compressed shifts and finite-rank operators of special kind. Recall that the compressed shift $S_{\alpha}$ is defined as $S_{\alpha}=A_{z}^{\alpha}=P_{\alpha} S_{\mid K_{\alpha}}$. As $K_{\alpha}$ is $S^{*}$-invariant, we have $S_{\alpha}^{*}=A_{\bar{z}}^{\alpha}=S_{\mid K_{\alpha}}^{*}$.

For all $n \in \mathbb{N}_{0}$ and $w \in \mathbb{D}$, the functional $f \mapsto f^{(n)}(w)$ is bounded on $H^{2}$, and so there exists $k_{w, n} \in H^{2}$ such that $f^{(n)}(w)=\left\langle f, k_{w, n}\right\rangle$. It is not difficult to verify that $k_{w, n}(z)=n ! z^{n} /(1-\bar{w} z)^{n+1}$ and, in particular, $k_{0, n}(z)=n ! z^{n}$. Denote $k_{w}(z)=k_{w, 0}(z)=1 /(1-\bar{w} z)$.

Lemma 2. Let $k \in \mathbb{N}$. For $f \in H^{2}$, we have

(a) $\left(S^{*}\right)^{k} f(z)=\bar{z}^{k} f(z)-\sum_{j=0}^{k-1}\left(\left\langle f, k_{0, j}\right\rangle / j !\right) \bar{z}^{k-j},|z|=1$;

(b) $W_{k}^{*} f-z^{k} W_{k}^{*} S^{*} f=\left\langle f, k_{0}\right\rangle=f(0)$.

Proof. Let $k \in \mathbb{N}$ and $f \in H^{2}$. Then $\left(S^{*}\right)^{k} f=\left(T_{\bar{z}}\right)^{k} f=P\left(\bar{z}^{k} f\right)$. Since $f(z)=\sum_{j=0}^{\infty}\left(\left\langle f, k_{0, j}\right\rangle / j !\right) z^{j}$, we have, for $|z|=1$,

$$
\begin{aligned}
\left(S^{*}\right)^{k} f(z) & =P\left(\sum_{j=0}^{\infty} \frac{\left\langle f, k_{0, j}\right\rangle}{j !} z^{j-k}\right)=\sum_{j=k}^{\infty} \frac{\left\langle f, k_{0, j}\right\rangle}{j !} z^{j-k} \\
& =\bar{z}^{k} f(z)-\sum_{j=0}^{k-1} \frac{\left\langle f, k_{0, j}\right\rangle}{j !} \bar{z}^{k-j} .
\end{aligned}
$$

Now observe that

$$
\begin{aligned}
W_{k}^{*} f(z)-z^{k} W_{k}^{*} S^{*} f(z) & =f\left(z^{k}\right)-z^{k} W_{k}^{*}(\bar{z} f(z)-\bar{z} f(0)) \\
& =f\left(z^{k}\right)-\left(f\left(z^{k}\right)-f(0)\right)=f(0),
\end{aligned}
$$

which proves (b).

As $K_{\alpha}$ is a closed subspace of $H^{2}, f \mapsto f^{(n)}(w)$ is also bounded on $K_{\alpha}$ for all $n \in \mathbb{N}_{0}$ and $w \in \mathbb{D}$. Then $f^{(n)}(w)=\left\langle f, k_{w, n}^{\alpha}\right\rangle$ for all $f \in K_{\alpha}$, where $k_{w, n}^{\alpha}=P_{\alpha} k_{w, n}$ (see, e.g., [12, p. 577]). In particular, $k_{w}^{\alpha}=P_{\alpha} k_{w, 0}$ 
is the reproducing kernel given by (1.1). Denote $\widetilde{k}_{w, n}^{\alpha}=C_{\alpha} k_{w, n}^{\alpha}$ and $\widetilde{k}_{w}^{\alpha}=\widetilde{k}_{w, 0}^{\alpha}$. Note that, a.e. on $\mathbb{T}$,

$$
\widetilde{k}_{w}^{\alpha}(z)=C_{\alpha} k_{w}^{\alpha}(z)=\frac{\alpha(z)-\alpha(w)}{z-w},
$$

and this formula is true also for all $z \in \mathbb{D} \backslash\{w\}$ (and we have $\widetilde{k}_{w}^{\alpha}(w)=\alpha^{\prime}(w)$ ).

Lemma 3. Let $k \in \mathbb{N}$ and $\varphi \in L^{2}$. Then

$$
U_{\varphi}^{\alpha, \beta}-S_{\beta} U_{\varphi}^{\alpha, \beta}\left(S_{\alpha}^{*}\right)^{k}=k_{0}^{\beta} \otimes \chi+\sum_{j=0}^{k-1} \psi_{j} \otimes k_{0, j}^{\alpha},
$$

where the equality holds on $K_{\alpha}^{\infty}$ with

$$
\chi=P_{\alpha}(\bar{\varphi}) \quad \text { and } \quad \psi_{j}=\frac{1}{j !} S_{\beta} P_{\beta} W_{k}\left(\varphi \bar{z}^{k-j}\right), \quad 0 \leqslant j \leqslant k-1 .
$$

Proof. Let $f \in K_{\alpha}^{\infty}$ and $g \in K_{\beta}^{\infty}$. Since $S_{\alpha}^{*}=P_{\alpha} S_{\mid K_{\alpha}}^{*}=S_{\mid K_{\alpha}}^{*}$ (as $K_{\alpha}$ is $S^{*}$-invariant), we have $S_{\alpha}^{*} f=S^{*} f$ and $S_{\beta}^{*} g=S^{*} g$ (note that here the functions $S^{*} f$ and $S^{*} g$ are bounded). By Lemma 2(a) we get

$$
\begin{aligned}
& \left\langle\left(U_{\varphi}^{\alpha, \beta}-S_{\beta} U_{\varphi}^{\alpha, \beta}\left(S_{\alpha}^{*}\right)^{k}\right) f, g\right\rangle \\
& \quad=\left\langle\varphi f, W_{k}^{*} g\right\rangle-\left\langle\varphi\left(S_{\alpha}^{*}\right)^{k} f, W_{k}^{*} S_{\beta}^{*} g\right\rangle=\left\langle\varphi f, W_{k}^{*} g\right\rangle-\left\langle\varphi\left(S^{*}\right)^{k} f, W_{k}^{*} S_{\beta}^{*} g\right\rangle \\
& =\left\langle\varphi f, W_{k}^{*} g\right\rangle-\left\langle\varphi \bar{z}^{k} f, W_{k}^{*} S_{\beta}^{*} g\right\rangle+\sum_{j=0}^{k-1} \frac{\left\langle f, k_{0, j}\right\rangle}{j !}\left\langle\varphi \bar{z}^{k-j}, W_{k}^{*} S_{\beta}^{*} g\right\rangle \\
& =\left\langle\varphi f, W_{k}^{*} g-z^{k} W_{k}^{*} S_{\beta}^{*} g\right\rangle+\sum_{j=0}^{k-1} \frac{1}{j !}\left\langle f, P_{\alpha} k_{0, j}\right\rangle\left\langle P_{\beta} W_{k}\left(\varphi \bar{z}^{k-j}\right), S_{\beta}^{*} g\right\rangle \\
& =\left\langle\varphi f, W_{k}^{*} g-z^{k} W_{k}^{*} S^{*} g\right\rangle+\sum_{j=0}^{k-1} \frac{1}{j !}\left\langle f, k_{0, j}^{\alpha}\right\rangle\left\langle S_{\beta} P_{\beta} W_{k}\left(\varphi \bar{z}^{k-j}\right), g\right\rangle .
\end{aligned}
$$

By Lemma 2(b) we have

$$
\left\langle\varphi f, W_{k}^{*} g-z^{k} W_{k}^{*} S^{*} g\right\rangle=\left\langle\varphi f,\left\langle g, k_{0}^{\beta}\right\rangle\right\rangle=\left\langle\left\langle f, P_{\alpha}(\bar{\varphi})\right\rangle k_{0}^{\beta}, g\right\rangle .
$$

For $k=2$, the following fact was partly noted in [18].

Lemma 4. Let $k \in \mathbb{N}, m \in \mathbb{Z}$, and let $j \in \mathbb{Z}$ be such that $|j|<k$. Then

$$
W_{k} M_{z^{m k+j}} W_{k}^{*}= \begin{cases}M_{z^{m}} & \text { if } j=0, \\ 0 & \text { if } 0<|j|<k .\end{cases}
$$

Proof. Let $f \in L^{2}, f=\sum_{n=-\infty}^{\infty} a_{n} z^{n}$. Then

$$
W_{k}^{*} f(z)=\sum_{n=-\infty}^{\infty} a_{n} z^{n k} \quad \text { and } \quad M_{z^{m k+j}} W_{k}^{*} f(z)=\sum_{n=-\infty}^{\infty} a_{n} z^{n k+m k+j} .
$$


If $0<|j|<k$, then $n k+m k+j$ is not divisible by $k$, and so $W_{k} M_{z^{m k+j}} W_{k}^{*} f=0$. On the other hand, if $j=0$, then

$$
W_{k} M_{z^{m k+j}} W_{k}^{*} f=W_{k}\left(\sum_{n=-\infty}^{\infty} a_{n} z^{n k+m k}\right)=\sum_{n=-\infty}^{\infty} a_{n} z^{n+m}=M_{z^{m}} f
$$

Lemma 5. Let $k \in \mathbb{N}, \chi \in K_{\alpha}$, and let $\psi_{0}, \ldots, \psi_{k-1} \in K_{\beta}$ be such that $\psi_{0}(0)=\cdots=\psi_{k-1}(0)=0$. Then for

$$
\varphi=\bar{\chi}+\sum_{j=1}^{k}(k-j) !\left(W_{k}^{*} S_{\beta}^{*} \psi_{k-j}\right) \cdot z^{j} \in L^{2},
$$

we have

$$
U_{\varphi}^{\alpha, \beta}-S_{\beta} U_{\varphi}^{\alpha, \beta}\left(S_{\alpha}^{*}\right)^{k}=k_{0}^{\beta} \otimes \chi+\sum_{j=0}^{k-1} \psi_{j} \otimes k_{0, j}^{\alpha} \quad \text { on } K_{\alpha}^{\infty}
$$

Proof. Note that for each $j \in\{1, \ldots, k\}$, we have

$$
\left(W_{k}^{*} S_{\beta}^{*} \psi_{k-j}\right)(z) \cdot z^{j}=\frac{\psi_{k-j}\left(z^{k}\right)}{z^{k}} z^{j} \in z H^{2}
$$

since $\psi_{k-j}(0)=0$. This and the fact that $P_{\alpha}=P_{\alpha} P$ give

$$
P_{\alpha}(\bar{\varphi})=P_{\alpha} P(\bar{\varphi})=P_{\alpha} \chi=\chi .
$$

Moreover, since $P_{\beta}=P_{\beta} P$ and $P W_{k}=W_{k} P$, for $1 \leqslant l \leqslant k$, we have

$$
\begin{aligned}
P_{\beta} W_{k}\left(\varphi \bar{z}^{l}\right) & =P_{\beta} W_{k}\left(\overline{\chi z^{l}}+\sum_{j=1}^{k}(k-j) !\left(W_{k}^{*} S_{\beta}^{*} \psi_{k-j}\right) \cdot z^{j-l}\right) \\
& =P_{\beta} P W_{k}\left(\overline{\chi z^{l}}\right)+P_{\beta} W_{k}\left(\sum_{j=1}^{k}(k-j) !\left(W_{k}^{*} S_{\beta}^{*} \psi_{k-j}\right) \cdot z^{j-l}\right) \\
& =P_{\beta} W_{k} P\left(\overline{\chi z^{l}}\right)+P_{\beta} W_{k}\left(\sum_{j=1}^{k}(k-j) ! M_{z^{j-l}} W_{k}^{*} S_{\beta}^{*} \psi_{k-j}\right) \\
& =P_{\beta}\left(\sum_{j=1}^{k}(k-j) ! W_{k} M_{z^{j-l}} W_{k}^{*} S_{\beta}^{*} \psi_{k-j}\right)=(k-l) ! S_{\beta}^{*} \psi_{k-l}
\end{aligned}
$$

where the last equality follows from Lemma 4 and the fact that $0<|j-l|<k$ for each $j \in\{1, \ldots, k\} \backslash\{l\}$. Hence, for $0 \leqslant j \leqslant k-1$,

$$
\frac{1}{j !} S_{\beta} P_{\beta} W_{k}\left(\varphi \bar{z}^{k-j}\right)=\frac{1}{j !} S_{\beta}\left(j ! S_{\beta}^{*} \psi_{j}\right)=S_{\beta} S_{\beta}^{*} \psi_{j}=\psi_{j} \quad\left(\psi_{j}(0)=0\right),
$$

and (3.1) follows from Lemma 3. 
Theorem 1. Let $U$ be a bounded linear operator from $K_{\alpha}$ into $K_{\beta}$. Then $U \in \mathcal{S}_{k}(\alpha, \beta), k \in \mathbb{N}$, if and only if there exist functions $\chi \in K_{\alpha}$ and $\psi_{0}, \ldots, \psi_{k-1} \in K_{\beta}$ such that

$$
U-S_{\beta} U\left(S_{\alpha}^{*}\right)^{k}=k_{0}^{\beta} \otimes \chi+\sum_{j=0}^{k-1} \psi_{j} \otimes k_{0, j}^{\alpha}
$$

Proof. If $U \in \mathcal{S}_{k}(\alpha, \beta)$, then $U=U_{\varphi}^{\alpha, \beta}$ for some $\varphi \in L^{2}$, and it satisfies (3.2) by Lemma 3.

Assume that $U$ satisfies (3.2) for some $\chi \in K_{\alpha}$ and $\psi_{0}, \ldots, \psi_{k-1} \in K_{\beta}$. Without any loss of generality, we can additionally assume that $\psi_{0}(0)=\cdots=\psi_{k-1}(0)=0$ (otherwise, we would replace $\psi_{j}$ and $\chi$ with

$$
\psi_{j}-\frac{\psi_{j}(0)}{\left\|k_{0}^{\beta}\right\|^{2}} k_{0}^{\beta} \quad \text { and } \quad \chi+\sum_{j=0}^{k-1} \frac{\overline{\psi_{j}(0)}}{\left\|k_{0}^{\beta}\right\|^{2}} k_{0, j}^{\alpha}
$$

respectively). By (3.2), for every $l \in \mathbb{N}_{0}$,

$$
S_{\beta}^{l} U\left(S_{\alpha}^{*}\right)^{k l}-S_{\beta}^{l+1} U\left(S_{\alpha}^{*}\right)^{(l+1) k}=S_{\beta}^{l} k_{0}^{\beta} \otimes S_{\alpha}^{k l} \chi+\sum_{j=0}^{k-1} S_{\beta}^{l} \psi_{j} \otimes S_{\alpha}^{k l} k_{0, j}^{\alpha} .
$$

It follows that for any $n \in \mathbb{N}$,

$$
U=\sum_{l=0}^{n}\left(S_{\beta}^{l} k_{0}^{\beta} \otimes S_{\alpha}^{k l} \chi+\sum_{j=0}^{k-1} S_{\beta}^{l} \psi_{j} \otimes S_{\alpha}^{k l} k_{0, j}^{\alpha}\right)+S_{\beta}^{n+1} U\left(S_{\alpha}^{*}\right)^{(n+1) k}
$$

Recall that for every $f=\sum_{n=0}^{\infty} a_{n} z^{n} \in H^{2}$, we have $\left\|\left(S^{*}\right)^{m} f\right\|^{2}=\sum_{n=m}^{\infty}\left|a_{n}\right|^{2} \rightarrow 0$ as $m \rightarrow \infty$. Hence for each $f \in K_{\alpha}$,

$$
0 \leqslant\left\|S_{\beta}^{n+1} U\left(S_{\alpha}^{*}\right)^{(n+1) k} f\right\| \leqslant\|U\| \cdot\left\|\left(S_{\alpha}^{*}\right)^{(n+1) k} f\right\| \rightarrow 0 \quad \text { as } n \rightarrow \infty
$$

and so

$$
U f=\sum_{l=0}^{\infty}\left(S_{\beta}^{l} k_{0}^{\beta} \otimes S_{\alpha}^{k l} \chi+\sum_{j=0}^{k-1} S_{\beta}^{l} \psi_{j} \otimes S_{\alpha}^{k l} k_{0, j}^{\alpha}\right) f
$$

Let now

$$
\varphi=\bar{\chi}+\psi \quad \text { with } \quad \psi=\sum_{j=1}^{k}(k-j) !\left(W_{k}^{*} S_{\beta}^{*} \psi_{k-j}\right) \cdot z^{j} \in H^{2} .
$$

By Lemma 5 we have

$$
U_{\varphi}^{\alpha, \beta}-S_{\beta} U_{\varphi}^{\alpha, \beta}\left(S_{\alpha}^{*}\right)^{k}=k_{0}^{\beta} \otimes \chi+\sum_{j=0}^{k-1} \psi_{j} \otimes k_{0, j}^{\alpha} \quad \text { on } K_{\alpha}^{\infty}
$$

and as above, (3.3) holds with $U_{\varphi}^{\alpha, \beta}$ in place of $U$ (on $K_{\alpha}^{\infty}$ ). For $f \in K_{\alpha}^{\infty}$ and $g \in K_{\beta}^{\infty}$, we have

$$
\left\langle S_{\beta}^{n+1} U_{\varphi}^{\alpha, \beta}\left(S_{\alpha}^{*}\right)^{(n+1) k} f, g\right\rangle=\left\langle\varphi\left(S_{\alpha}^{*}\right)^{k(n+1)} f, W_{k}^{*}\left(S_{\beta}^{*}\right)^{n+1} g\right\rangle \rightarrow 0 \quad \text { as } n \rightarrow \infty \text {. }
$$


Indeed, since

$$
\begin{aligned}
\langle\varphi & \left.\left(S_{\alpha}^{*}\right)^{k(n+1)} f, W_{k}^{*}\left(S_{\beta}^{*}\right)^{n+1} g\right\rangle \\
& =\left\langle\varphi\left(S^{*}\right)^{k(n+1)} f, W_{k}^{*}\left(S^{*}\right)^{n+1} g\right\rangle \\
& =\left\langle\bar{\chi} \cdot T_{\bar{z}^{k(n+1)}} f, W_{k}^{*}\left(S^{*}\right)^{n+1} g\right\rangle+\left\langle\psi \cdot\left(S^{*}\right)^{k(n+1)} f, W_{k}^{*} T_{\bar{z}^{n+1}} g\right\rangle \\
& =\left\langle P\left(\bar{z}^{k(n+1)} f\right), \chi \cdot W_{k}^{*}\left(S^{*}\right)^{n+1} g\right\rangle+\left\langle\psi \cdot\left(S^{*}\right)^{k(n+1)} f, W_{k}^{*} P\left(\bar{z}^{n+1} g\right)\right\rangle \\
& =\left\langle\bar{z}^{k(n+1)} f, \chi \cdot W_{k}^{*}\left(S^{*}\right)^{n+1} g\right\rangle+\left\langle\psi \cdot\left(S^{*}\right)^{k(n+1)} f, P W_{k}^{*}\left(\bar{z}^{n+1} g\right)\right\rangle \\
& =\left\langle\overline{\chi z^{k(n+1)}} f, W_{k}^{*}\left(S^{*}\right)^{n+1} g\right\rangle+\left\langle\psi \cdot\left(S^{*}\right)^{k(n+1)} f, \bar{z}^{k(n+1)} \cdot W_{k}^{*} g\right\rangle \\
& =\left\langle P\left(\overline{\chi z^{k(n+1)}} f\right), W_{k}^{*}\left(S^{*}\right)^{n+1} g\right\rangle+\left\langle\left(S^{*}\right)^{k(n+1)} f, P\left(\overline{\psi z^{k(n+1)}} \cdot W_{k}^{*} g\right)\right\rangle,
\end{aligned}
$$

condition (3.5) follows from the fact that

$$
\left|\left\langle P\left(\overline{\chi z^{k(n+1)}} f\right), W_{k}^{*}\left(S^{*}\right)^{n+1} g\right\rangle\right| \leqslant\|\chi f\| \cdot\left\|\left(S^{*}\right)^{n+1} g\right\| \rightarrow 0 \quad \text { as } n \rightarrow \infty,
$$

and

$$
\left|\left\langle\left(S^{*}\right)^{k(n+1)} f, P \overline{\overline{\psi z^{k(n+1)}}} \cdot W_{k}^{*} g\right)\right\rangle \mid \leqslant\left\|\left(S^{*}\right)^{k(n+1)} f\right\| \cdot\left\|\psi \cdot W_{k}^{*} g\right\| \rightarrow 0 \quad \text { as } n \rightarrow \infty .
$$

This means that for $f \in K_{\alpha}^{\infty}$ and $g \in K_{\beta}^{\infty}$,

$$
\left\langle U_{\varphi}^{\alpha, \beta} f, g\right\rangle=\left\langle\sum_{l=0}^{\infty}\left(S_{\beta}^{l} k_{0}^{\beta} \otimes S_{\alpha}^{k l} \chi+\sum_{j=0}^{k-1} S_{\beta}^{l} \psi_{j} \otimes S_{\alpha}^{k l} k_{0, j}^{\alpha}\right) f, g\right\rangle,
$$

and by (3.4) we have

$$
\left\langle U_{\varphi}^{\alpha, \beta} f, g\right\rangle=\langle U f, g\rangle .
$$

It follows that $U=U_{\varphi}^{\alpha, \beta} \in \mathcal{S}_{k}(\alpha, \beta)$.

Corollary 4. If a bounded linear operator $U: K_{\alpha} \rightarrow K_{\beta}$ satisfies (3.2), then $U=U_{\varphi}^{\alpha, \beta}$ with

$$
\varphi=\bar{\chi}+\sum_{j=0}^{k-1} \psi_{j}\left(z^{k}\right) j ! \bar{z}^{j}
$$

Proof. Assume that $U$ satisfies (3.2). If $\psi_{0}(0)=\cdots=\psi_{k-1}(0)=0$, then following the proof of Theorem 1, we get that $U=U_{\varphi}^{\alpha, \beta}$ with

$$
\varphi=\bar{\chi}+\sum_{j=0}^{k-1} j !\left(W_{k}^{*} S_{\beta}^{*} \psi_{j}\right) \cdot z^{k-j}=\bar{\chi}+\sum_{j=0}^{k-1} \bar{z}^{k} \psi_{j}\left(z^{k}\right) j ! z^{k-j}=\bar{\chi}+\sum_{j=0}^{k-1} \psi_{j}\left(z^{k}\right) j ! \bar{z}^{j} .
$$

Otherwise, as explained at the beginning of the proof of Theorem 1, replace $\psi_{j}$ and $\chi$ with

$$
\psi_{j}-\frac{\psi_{j}(0)}{\left\|k_{0}^{\beta}\right\|^{2}} k_{0}^{\beta} \quad \text { and } \quad \chi+\sum_{j=0}^{k-1} \frac{\overline{\psi_{j}(0)}}{\left\|k_{0}^{\beta}\right\|^{2}} k_{0, j}^{\alpha},
$$


respectively. Then, by the first part of the proof, $U=U_{\varphi_{1}}^{\alpha, \beta}$ with

$$
\varphi_{1}=\overline{\left(\chi+\sum_{j=0}^{k-1} \frac{\overline{\psi_{j}(0)}}{\left\|k_{0}^{\beta}\right\|^{2}} k_{0, j}^{\alpha}\right)}+\sum_{j=0}^{k-1}\left(\psi_{j}\left(z^{k}\right)-\frac{\psi_{j}(0)}{\left\|k_{0}^{\beta}\right\|^{2}} k_{0}^{\beta}\left(z^{k}\right)\right) j ! \bar{z}^{j} .
$$

However, in that case,

$$
\begin{aligned}
& \varphi_{1}-\varphi=\sum_{j=0}^{k-1} \frac{\psi_{j}(0)}{\left\|k_{0}^{\beta}\right\|^{2}} \overline{k_{0, j}^{\alpha}}-\sum_{j=0}^{k-1} \frac{\psi_{j}(0)}{\left\|k_{0}^{\beta}\right\|^{2}}\left(1-\overline{\beta(0)} \beta\left(z^{k}\right)\right) j ! \bar{z}^{j} \\
& =\sum_{j=0}^{k-1} \frac{\psi_{j}(0)}{\left\|k_{0}^{\beta}\right\|^{2}} \overline{P_{\alpha}\left(j ! z^{j}\right)}-\sum_{j=0}^{k-1} \frac{\psi_{j}(0)}{\left\|k_{0}^{\beta}\right\|^{2}} j ! \bar{z}^{j}+\overline{\beta(0)} \beta\left(z^{k}\right) \sum_{j=0}^{k-1} \frac{\psi_{j}(0)}{\left\|k_{0}^{\beta}\right\|^{2}} j ! \bar{z}^{j} \\
& =\overline{\left(P_{\alpha}\left(\sum_{j=0}^{k-1} \overline{\frac{\psi_{j}(0)}{\left\|k_{0}^{\beta}\right\|^{2}}} j ! z^{j}\right)-\sum_{j=0}^{k-1} \overline{\frac{\psi_{j}(0)}{\left\|k_{0}^{\beta}\right\|^{2}}} j ! z^{j}\right)}+\bar{z}^{k-1} W_{k}^{*} \beta \cdot \overline{\beta(0)} \sum_{j=0}^{k-1} \frac{\psi_{j}(0)}{\left\|k_{0}^{\beta}\right\|^{2}} j ! z^{k-1-j} \\
& \in \overline{\alpha H^{2}}+\bar{z}^{k-1}\left(W_{k}^{*} \beta\right) H^{2} \text {, }
\end{aligned}
$$

and so, by Proposition 3, $U=U_{\varphi_{1}}^{\alpha, \beta}=U_{\varphi}^{\alpha, \beta}$.

Note that if $\psi_{0}(0)=\cdots=\psi_{k-1}(0)=0$, then the pairwise orthogonal functions $\psi_{j}\left(z^{k}\right) j ! \bar{z}^{j}, 0 \leqslant j \leqslant k-1$, all belong to $z H^{2}$.

Corollary 5. If $U \in \mathcal{S}_{k}(\alpha, \beta), k \in \mathbb{N}$, then there exist functions $\chi \in K_{\alpha}$ and $\psi_{0}, \ldots, \psi_{k-1} \in K_{\beta}$ such that $U=U_{\varphi}^{\alpha, \beta}$ with

$$
\varphi=\bar{\chi}+\psi_{0}\left(z^{k}\right)+\frac{1}{z} \psi_{1}\left(z^{k}\right)+\frac{1}{z^{2}} \psi_{2}\left(z^{k}\right)+\cdots+\frac{1}{z^{k-1}} \psi_{k-1}\left(z^{k}\right)
$$

and $\psi_{0}(0)=\cdots=\psi_{k-1}(0)=0$. Moreover, this decomposition is orthogonal.

Corollary 6. Let $U$ be a bounded linear operator from $K_{\alpha}$ into $K_{\beta}$. Then $U \in \mathcal{S}_{k}(\alpha, \beta), k \in \mathbb{N}$, if and only if there exist functions $\chi \in K_{\alpha}$ and $\psi_{0}, \ldots, \psi_{k-1} \in K_{\beta}$ such that

$$
U-S_{\beta}^{*} U S_{\alpha}^{k}=\widetilde{k}_{0}^{\beta} \otimes \chi+\sum_{j=0}^{k-1} \psi_{j} \otimes \widetilde{k}_{0, j}^{\alpha}
$$

Proof. By Corollary 2, $U \in \mathcal{S}_{k}(\alpha, \beta)$ if and only if $C_{\beta} U C_{\alpha} \in \mathcal{S}_{k}(\alpha, \beta)$. By Theorem 1 the latter happens if and only if

$$
C_{\beta} U C_{\alpha}-S_{\beta}\left(C_{\beta} U C_{\alpha}\right)\left(S_{\alpha}^{*}\right)^{k}=k_{0}^{\beta} \otimes \mu+\sum_{j=0}^{k-1} \nu_{j} \otimes k_{0, j}^{\alpha}
$$

for some functions $\mu \in K_{\alpha}$ and $\nu_{0}, \ldots, \nu_{k-1} \in K_{\beta}$. Since $C_{\alpha}$ is an involution, (3.7) is equivalent to

$$
C_{\beta}^{2} U C_{\alpha}^{2}-C_{\beta} S_{\beta} C_{\beta} U C_{\alpha}\left(S_{\alpha}^{*}\right)^{k} C_{\alpha}=C_{\beta}\left(k_{0}^{\beta} \otimes \mu\right) C_{\alpha}+\sum_{j=0}^{k-1} C_{\beta}\left(\nu_{j} \otimes k_{0, j}^{\alpha}\right) C_{\alpha}
$$


By $C_{\alpha}$-symmetry of the compressed shift, this can be written as

$$
U-S_{\beta}^{*} U S_{\alpha}^{k}=\widetilde{k}_{0}^{\beta} \otimes \chi+\sum_{j=0}^{k-1} \psi_{j} \otimes \widetilde{k}_{0, j}^{\alpha}
$$

with $\chi=C_{\alpha} \mu \in K_{\alpha}$ and $\psi_{j}=C_{\beta} \nu_{j} \in K_{\beta}$ for $j=0,1, \ldots, k-1$.

Corollary 7. If a bounded linear operator $U: K_{\alpha} \rightarrow K_{\beta}$ satisfies (3.6), then

$$
U=U_{\varphi}^{\alpha, \beta} \quad \text { with } \varphi=\beta\left(z^{k}\right) \bar{z}^{k} \bar{\chi}+\bar{\alpha} \sum_{j=0}^{k-1} \psi_{j}\left(z^{k}\right) j ! z^{j+1} .
$$

Proof. Assume that $U$ satisfies (3.6). A reasoning similar to that given in the proof of Corollary 6 shows that $C_{\beta} U C_{\alpha}$ satisfies (3.7) with $\mu=C_{\alpha} \chi$ and $\nu_{j}=C_{\beta} \psi_{j}$. By Corollary $4, C_{\beta} U C_{\alpha}=U_{\psi}^{\alpha, \beta}$ with $\psi=$ $\bar{\mu}+\sum_{j=0}^{k-1} \nu_{j}\left(z^{k}\right) j ! \bar{z}^{j}$, and by Proposition $2, U=C_{\beta} U_{\psi}^{\alpha, \beta} C_{\alpha}=U_{\varphi}^{\alpha, \beta}$ with

$$
\begin{aligned}
\varphi & =\overline{z^{k-1} \alpha \psi} W_{k}^{*} \beta=W_{k}^{*} \beta\left(\overline{z^{k-1} \alpha} \mu+\overline{z^{k-1} \alpha} \sum_{j=0}^{k-1} \overline{\nu_{j}\left(z^{k}\right)} j ! z^{j}\right) \\
& =\beta\left(z^{k}\right)\left(\overline{z^{k-1} \alpha} \alpha \overline{z \chi}+\sum_{j=0}^{k-1} \overline{z^{k-1} \alpha} \overline{\beta\left(z^{k}\right)} z^{k} \psi_{j}\left(z^{k}\right) j ! z^{j}\right) \\
& =\beta\left(z^{k}\right) \bar{z}^{k} \bar{\chi}+\sum_{j=0}^{k-1} \bar{\alpha} \psi_{j}\left(z^{k}\right) j ! z^{j+1} .
\end{aligned}
$$

Using Theorem 1 and Corollary 6, we can prove the following:

Corollary 8. Let $U$ be a bounded linear operator from $K_{\alpha}$ into $K_{\beta}$. Then $U \in \mathcal{S}_{k}(\alpha, \beta), k \in \mathbb{N}$, if and only if there exist functions $\chi \in K_{\alpha}$ and $\psi_{0}, \ldots, \psi_{k-1} \in K_{\beta}$ (possibly different for different conditions) such that one (and all) of the following conditions holds:

(a) $S_{\beta}^{*} U-U\left(S_{\alpha}^{*}\right)^{k}=\widetilde{k}_{0}^{\beta} \otimes \chi+\sum_{j=0}^{k-1} \psi_{j} \otimes k_{0, j}^{\alpha}$,

(b) $S_{\beta} U-U S_{\alpha}^{k}=k_{0}^{\beta} \otimes \chi+\sum_{j=0}^{k-1} \psi_{j} \otimes \widetilde{k}_{0, j}^{\alpha}$.

Proof. Use reasoning analogous to that presented in the proof of [17, Cor. 2.4].

Note that if $\operatorname{dim} K_{\beta}=1$, then by Theorem 1 all bounded linear operators from $K_{\alpha}$ into $K_{\beta}$ belong to $\mathcal{S}_{k}(\alpha, \beta)$ for each $k \in \mathbb{N}$.

Corollary 9. Let $\alpha$ and $\beta$ be two nonconstant inner functions and assume that $\operatorname{dim} K_{\alpha}=m<+\infty$. If $k \geqslant m$, then every bounded linear operator from $K_{\alpha}$ into $K_{\beta}$ belongs to $\mathcal{S}_{k}(\alpha, \beta)$.

Proof. Let $U$ be a bounded linear operator from $K_{\alpha}$ into $K_{\beta}$. If $\operatorname{dim} K_{\alpha}=m<+\infty$, then $U-S_{\beta} U\left(S_{\alpha}^{*}\right)^{k}$ has rank at most $m$, which by assumption is less than or equal to $k$. Hence there exist functions $g_{0}, \ldots, g_{k-1} \in$ $K_{\alpha}$ and $f_{0}, \ldots, f_{k-1} \in K_{\beta}$ (some of these functions possibly equal to zero) such that $U-S_{\beta} U\left(S_{\alpha}^{*}\right)^{k}=$ $\sum_{j=0}^{k-1} f_{j} \otimes g_{j}$. Since here the kernels $k_{0,0}^{\alpha}, k_{0,1}^{\alpha}, \ldots, k_{0, m-1}^{\alpha}$ are linearly independent and span $K_{\alpha}$, each $g_{j}$ can be written as a linear combination of these kernels, and so $U$ satisfies (3.2). 
Example 2. As in Example 1, consider $\alpha(z)=z^{4}$ and $\beta(z)=z^{3}$. Then, for $k=5$ and $\varphi=\sum_{n=-\infty}^{\infty} a_{n} z^{n} \in L^{2}$, the operator $U_{\varphi}^{\alpha, \beta}$ is represented by the matrix

$$
\left[\begin{array}{cccc}
a_{0} & a_{-1} & a_{-2} & a_{-3} \\
a_{5} & a_{4} & a_{3} & a_{2} \\
a_{10} & a_{9} & a_{8} & a_{7}
\end{array}\right] .
$$

It follows easily that every bounded linear operator from $K_{\alpha}$ into $K_{\beta}$ belongs to $\mathcal{S}_{5}(\alpha, \beta)$. Note that here $U_{z}^{\alpha, \beta}=0$, but

$$
z \notin \overline{\alpha H^{2}}+\bar{z}^{k-1}\left(W_{k}^{*} \beta\right) H^{2}=\overline{z^{4} H^{2}}+z^{11} H^{2} .
$$

Finally, we use Theorem 1 to describe some rank-one operators from $\mathcal{S}_{k}(\alpha, \beta)$.

Proposition 4. Let $\alpha$, $\beta$ be two nonconstant inner functions, and let $k \in \mathbb{N}$. Then for each $l \in\{0,1, \ldots, k-1\}$, the rank-one operators $\widetilde{k}_{0}^{\beta} \otimes k_{0, l}^{\alpha}$ and $k_{0}^{\beta} \otimes \widetilde{k}_{0, l}^{\alpha}$ belong to $\mathcal{S}_{k}(\alpha, \beta)$.

Proof. Let $l \in\{0,1, \ldots, k-1\}$. Since $S_{\beta} \widetilde{k}_{0}^{\beta}=P_{\beta}(\beta-\beta(0))=-\beta(0) P_{\beta} 1=-\beta(0) k_{0}^{\beta}$ (see [29, Lemma 2.2(a)]), we have

$$
\begin{aligned}
\widetilde{k}_{0}^{\beta} \otimes k_{0, l}^{\alpha}-S_{\beta}\left(\widetilde{k}_{0}^{\beta} \otimes k_{0, l}^{\alpha}\right)\left(S_{\alpha}^{*}\right)^{k} & =\widetilde{k}_{0}^{\beta} \otimes k_{0, l}^{\alpha}-\left(S_{\beta} \widetilde{k}_{0}^{\beta}\right) \otimes\left(S_{\alpha}^{k} k_{0, l}^{\alpha}\right) \\
& =\widetilde{k}_{0}^{\beta} \otimes k_{0, l}^{\alpha}+\left(\beta(0) k_{0}^{\beta}\right) \otimes\left(S_{\alpha}^{k} k_{0, l}^{\alpha}\right) \\
& =k_{0}^{\beta} \otimes\left(\overline{\beta(0)} S_{\alpha}^{k} k_{0, l}^{\alpha}\right)+\widetilde{k}_{0}^{\beta} \otimes k_{0, l}^{\alpha},
\end{aligned}
$$

and by Theorem 1, $\widetilde{k}_{0}^{\beta} \otimes k_{0, l}^{\alpha} \in \mathcal{S}_{k}(\alpha, \beta)$ since it satisfies (3.2) with $\chi=\overline{\beta(0)}\left(S_{\alpha}^{k} k_{0, l}^{\alpha}\right), \psi_{l}=\widetilde{k}_{0}^{\beta}$ and $\psi_{j}=0$ for $j \neq l$. By Corollary 2 it follows that

$$
k_{0}^{\beta} \otimes \widetilde{k}_{0, l}^{\alpha}=C_{\beta}\left(\widetilde{k}_{0}^{\beta} \otimes k_{0, l}^{\alpha}\right) C_{\alpha} \in \mathcal{S}_{k}(\alpha, \beta) .
$$

Corollary 10. For each $l \in\{0,1, \ldots, k-1\}$,

(a) $\widetilde{k}_{0}^{\beta} \otimes k_{0, l}^{\alpha}=U_{\varphi}^{\alpha, \beta}$ with $\varphi=W_{k}^{*} \beta \cdot l ! \bar{z}^{l+k}$,

(b) $k_{0}^{\beta} \otimes \widetilde{k}_{0, l}^{\alpha}=U_{\psi}^{\alpha, \beta}$ with $\psi=\bar{\alpha} \cdot l ! z^{l+1}$.

Proof. By Corollary 4 and the proof of Proposition $4, \widetilde{k}_{0}^{\beta} \otimes k_{0, l}^{\alpha}=U_{\varphi_{1}}^{\alpha, \beta}$ with

$$
\varphi_{1}=\beta(0) \overline{\left(S_{\alpha}^{k} k_{0, l}^{\alpha}\right)}+\widetilde{k}_{0}^{\beta}\left(z^{k}\right) l ! \bar{z}^{l} .
$$

Since for each $g \in K_{\alpha}$,

$$
\left\langle S_{\alpha}^{k} k_{0, l}^{\alpha}, g\right\rangle=\left\langle P_{\alpha}\left(l ! z^{l}\right),\left(S_{\alpha}^{*}\right)^{k} g\right\rangle=\left\langle l ! z^{l}, T_{\bar{z}^{k}} g\right\rangle=\left\langle l ! z^{l+k}, g\right\rangle,
$$

we have $S_{\alpha}^{k} k_{0, l}^{\alpha}=P_{\alpha}\left(l ! z^{l+k}\right)$ and

$$
\varphi_{1}=\beta(0) \overline{P_{\alpha}\left(l ! z^{l+k}\right)}+\left(\beta\left(z^{k}\right)-\beta(0)\right) l ! \bar{z}^{l+k}=W_{k}^{*} \beta \cdot l ! \bar{z}^{l+k}-\beta(0) \overline{\left(l ! z^{l+k}-P_{\alpha}\left(l ! z^{l+k}\right)\right)} .
$$

Since $\varphi_{1}-\varphi \in \overline{\alpha H^{2}}$, we get $\widetilde{k}_{0}^{\beta} \otimes k_{0, l}^{\alpha}=U_{\varphi_{1}}^{\alpha, \beta}=U_{\varphi}^{\alpha, \beta}$, that is, (a) holds.

Now (b) follows from Proposition 2. 


\section{Operators from $\mathcal{S}_{k}(\alpha, \beta)$ equal to the zero operator, part 2}

Let $\alpha, \beta$ be two nonconstant inner functions. In this section, we completely determine which $\varphi \in L^{2}$ give $U_{\varphi}^{\alpha, \beta}=0$.

Note first that from Lemma 3 we can obtain the following corollary.

Corollary 11. Let $k \in \mathbb{N}$ and $\varphi \in \overline{H^{2}}$. Then $U_{\varphi}^{\alpha, \beta}=0$ if and only if $\varphi \in \overline{\alpha H^{2}}$.

Proof. If $\varphi \in \overline{\alpha H^{2}}$, then $U_{\varphi}^{\alpha, \beta}=0$ by Proposition 1 .

On the other hand, if $U_{\varphi}^{\alpha, \beta}=0$ for $\varphi \in \overline{H^{2}}$, then by Lemma 3

$$
0=U_{\varphi}^{\alpha, \beta}-S_{\beta} U_{\varphi}^{\alpha, \beta}\left(S_{\alpha}^{*}\right)^{k}=k_{0}^{\beta} \otimes \chi+\sum_{j=0}^{k-1} \psi_{j} \otimes k_{0, j}^{\alpha},
$$

where $\chi=P_{\alpha}(\bar{\varphi})$ and $\psi_{j}=(1 / j !) S_{\beta} P_{\beta} W_{k}\left(\varphi \bar{z}^{k-j}\right), 0 \leqslant j \leqslant k-1$. Since here $\varphi \cdot \bar{z}^{k-j} \in \overline{z H^{2}}$ for each $0 \leqslant j \leqslant k-1$,

$$
P_{\beta} W_{k}\left(\varphi \bar{z}^{k-j}\right)=P_{\beta} P W_{k}\left(\varphi \bar{z}^{k-j}\right)=P_{\beta} W_{k} P\left(\varphi \bar{z}^{k-j}\right)=0,
$$

and so we have $\psi_{0}=\psi_{1}=\cdots=\psi_{k-1}=0$. Hence $\chi=P_{\alpha}(\bar{\varphi})=0$, which means that $\bar{\varphi} \in \alpha H^{2}$, that is, $\varphi \in \overline{\alpha H^{2}}$.

Let us now consider analytic symbols. Observe that for a fixed $k \in \mathbb{N}$, each $\varphi \in H^{2}$ can be written as

$$
\varphi=W_{k}^{*} W_{k} \varphi+\bar{z} W_{k}^{*} W_{k}(z \varphi)+\cdots+\bar{z}^{k-1} W_{k}^{*} W_{k}\left(z^{k-1} \varphi\right)=\sum_{j=0}^{k-1} \bar{z}^{j} W_{k}^{*} W_{k}\left(z^{j} \varphi\right) .
$$

Indeed, if $\varphi=\sum_{n=0}^{\infty} a_{n} z^{n}$, then for each $j \in\{0,1, \ldots, k-1\}$,

$$
W_{k}\left(z^{j} \varphi\right)=W_{k}\left(\sum_{n=0}^{\infty} a_{n} z^{n+j}\right)= \begin{cases}a_{0}+\sum_{m=1}^{\infty} a_{m k} z^{m} & \text { for } j=0 \\ \sum_{m=1}^{\infty} a_{m k-j} z^{m} & \text { for } j>0 .\end{cases}
$$

Hence, for each $0<j \leqslant k-1$,

$$
\bar{z}^{j} W_{k}^{*} W_{k}\left(z^{j} \varphi\right)=\bar{z}^{j} W_{k}^{*}\left(\sum_{m=1}^{\infty} a_{m k-j} z^{m}\right)=\sum_{m=1}^{\infty} a_{m k-j} z^{m k-j}
$$

and

$$
W_{k}^{*} W_{k}(\varphi)=W_{k}^{*}\left(a_{0}+\sum_{m=1}^{\infty} a_{m k} z^{m}\right)=a_{0}+\sum_{m=1}^{\infty} a_{m k} z^{m k}
$$

Thus

$$
\begin{aligned}
\varphi & =\sum_{n=0}^{\infty} a_{n} z^{n}=a_{0}+\sum_{m=1}^{\infty} \sum_{j=0}^{k-1} a_{m k-j} z^{m k-j}=a_{0}+\sum_{m=1}^{\infty} a_{m k} z^{m k}+\sum_{j=1}^{k-1} \sum_{m=1}^{\infty} a_{m k-j} z^{m k-j} \\
& =W_{k}^{*} W_{k}(\varphi)+\sum_{j=1}^{k-1} \bar{z}^{j} W_{k}^{*} W_{k}\left(z^{j} \varphi\right) .
\end{aligned}
$$


Hence, if $\varphi \in H^{2}$, then

$$
\varphi=W_{k}^{*} \varphi_{0}+\bar{z} W_{k}^{*} \varphi_{1}+\cdots+\bar{z}^{k-1} W_{k}^{*} \varphi_{k-1}
$$

with $\varphi_{j}=W_{k}\left(z^{j} \varphi\right) \in H^{2}$. Note that $\varphi_{j} \in z H^{2}$ for $j>0$ and the above decomposition is orthogonal. Moreover, for arbitrary $\varphi_{0} \in H^{2}$ and $\varphi_{1}, \ldots, \varphi_{k-1} \in z H^{2}$, the function $\varphi$ given by (4.1) belongs to $H^{2}$ with the decomposition again orthogonal. It follows that

$$
H^{2}=W_{k}^{*} H^{2} \oplus \bar{z} W_{k}^{*}\left(z H^{2}\right) \oplus \cdots \oplus \bar{z}^{k-1} W_{k}^{*}\left(z H^{2}\right)
$$

and

$$
z H^{2}=W_{k}^{*}\left(z H^{2}\right) \oplus \bar{z} W_{k}^{*}\left(z H^{2}\right) \oplus \cdots \oplus \bar{z}^{k-1} W_{k}^{*}\left(z H^{2}\right) .
$$

As $W_{k}^{*}\left(z H^{2}\right)=z^{k} W_{k}^{*} H^{2}$, we also have

$$
H^{2}=W_{k}^{*} H^{2} \oplus z W_{k}^{*} H^{2} \oplus \cdots \oplus z^{k-1} W_{k}^{*} H^{2},
$$

and so

$$
z H^{2}=z W_{k}^{*} H^{2} \oplus z^{2} W_{k}^{*} H^{2} \oplus \cdots \oplus z^{k} W_{k}^{*} H^{2} .
$$

Now for any inner function $\beta$, by (4.2) we have

$$
\left(W_{k}^{*} \beta\right) \cdot H^{2}=W_{k}^{*}\left(\beta H^{2}\right) \oplus z W_{k}^{*}\left(\beta H^{2}\right) \oplus \cdots \oplus z^{k-1} W_{k}^{*}\left(\beta H^{2}\right) .
$$

Since $W_{k}^{*}$ is an isometry, we obtain

$$
K_{W_{k}^{*} \beta}=W_{k}^{*}\left(K_{\beta}\right) \oplus z W_{k}^{*}\left(K_{\beta}\right) \oplus \cdots \oplus z^{k-1} W_{k}^{*}\left(K_{\beta}\right) .
$$

We are now ready to prove the following:

Theorem 2. Let $\alpha, \beta$ be two nonconstant inner functions, let $k \in \mathbb{N}$, and assume that $\operatorname{dim} K_{\alpha} \geqslant k$. Moreover, let $\varphi \in L^{2}$. Then $U_{\varphi}^{\alpha, \beta}=0$ if and only if

$$
\varphi \in \overline{\alpha H^{2}}+\bar{z}^{k-1}\left(W_{k}^{*} \beta\right) H^{2} .
$$

Proof. If $\varphi \in \overline{\alpha H^{2}}+\bar{z}^{k-1}\left(W_{k}^{*} \beta\right) H^{2}$, then $U_{\varphi}^{\alpha, \beta}=0$ by Proposition 3 .

Assume that $\varphi \in L^{2}$ and $U_{\varphi}^{\alpha, \beta}=0$. Since

$$
L^{2}=\overline{H^{2}} \oplus z H^{2}=\overline{K_{\alpha}} \oplus \overline{\alpha H^{2}} \oplus\left(W_{k}^{*} \beta\right) z H^{2} \oplus z K_{W_{k}^{*} \beta},
$$

we can write

$$
\varphi=\bar{\varphi}_{1}+\overline{\alpha h_{1}}+\left(W_{k}^{*} \beta\right) \cdot z h_{2}+z \varphi_{2},
$$

where $\varphi_{1} \in K_{\alpha}, \varphi_{2} \in K_{W_{k}^{*} \beta}$ and $h_{1}, h_{2} \in H^{2}$. Since

$$
\left(W_{k}^{*} \beta\right) \cdot z h_{2}=\bar{z}^{k-1}\left(W_{k}^{*} \beta\right) \cdot z^{k} h_{2} \in \bar{z}^{k-1}\left(W_{k}^{*} \beta\right) H^{2},
$$


by the first part of the proof (or by Proposition 3) we get $0=U_{\varphi}^{\alpha, \beta}=U_{\bar{\varphi}_{1}+z \varphi_{2}}^{\alpha, \beta}$. It follows from Lemma 3 that

$$
0=U_{\bar{\varphi}_{1}+z \varphi_{2}}^{\alpha, \beta}-S_{\beta} U_{\bar{\varphi}_{1}+z \varphi_{2}}^{\alpha, \beta}\left(S_{\alpha}^{*}\right)^{k}=k_{0}^{\beta} \otimes \chi+\sum_{j=0}^{k-1} \psi_{j} \otimes k_{0, j}^{\alpha}
$$

with

$$
\chi=P_{\alpha}\left(\overline{\bar{\varphi}_{1}+z \varphi_{2}}\right)=P_{\alpha} \varphi_{1}=\varphi_{1}
$$

and

$$
\psi_{j}=\frac{1}{j !} S_{\beta} P_{\beta} W_{k}\left(\varphi \bar{z}^{k-j}\right)=\frac{1}{j !} S_{\beta} P_{\beta} W_{k}\left(\bar{\varphi}_{1} \bar{z}^{k-j}+\bar{z}^{k-j-1} \varphi_{2}\right)=\frac{1}{j !} S_{\beta} P_{\beta} W_{k}\left(\bar{z}^{k-j-1} \varphi_{2}\right)
$$

for $0 \leqslant j \leqslant k-1$. The last equality follows from the fact that

$$
P_{\beta} W_{k}\left(\bar{\varphi}_{1} \bar{z}^{k-j}\right)=W_{k} P_{W_{k}^{*} \beta}\left(\bar{\varphi}_{1} \bar{z}^{k-j}\right)=0 .
$$

Now by (4.4) we have

$$
\varphi_{2}=W_{k}^{*} f_{1}+z W_{k}^{*} f_{2}+\cdots+z^{k-1} W_{k}^{*} f_{k}
$$

for some $f_{1}, f_{2}, \ldots, f_{k} \in K_{\beta}$, and using Lemma 4 , we get

$$
\begin{aligned}
\psi_{j} & =\frac{1}{j !} S_{\beta} P_{\beta} W_{k}\left(z^{j-k+1} \sum_{l=1}^{k} z^{l-1} W_{k}^{*} f_{l}\right)=\frac{1}{j !} \sum_{l=1}^{k} S_{\beta} P_{\beta} W_{k} M_{z^{j-2 k+l}} W_{k}^{*}\left(z f_{l}\right) \\
& =\frac{1}{j !} S_{\beta} P_{\beta} W_{k} M_{z^{-k}} W_{k}^{*}\left(z f_{k-j}\right)=\frac{1}{j !} S_{\beta} P_{\beta}\left(\bar{z} z f_{k-j}\right)=\frac{1}{j !} S_{\beta}\left(f_{k-j}\right)
\end{aligned}
$$

$(|j+l-k| \leqslant k-1)$. Hence

$$
k_{0}^{\beta} \otimes \varphi_{1}+\sum_{j=0}^{k-1} \frac{1}{j !} S_{\beta}\left(f_{k-j}\right) \otimes k_{0, j}^{\alpha}=0 .
$$

Since $\operatorname{dim} K_{\alpha} \geqslant k$, the functions $k_{0, j}^{\alpha}, j=0, \ldots, k-1$, are linearly independent, which by (4.6) means that there exists constants $c_{0}, \ldots, c_{k-1} \in \mathbb{C}$ such that

$$
S_{\beta}\left(f_{k-j}\right)=c_{j} \cdot k_{0}^{\beta} \quad \text { for each } 0 \leqslant j \leqslant k-1
$$

and

$$
\varphi_{1}=-\sum_{j=0}^{k-1} \frac{1}{j !} \bar{c}_{j} \cdot k_{0, j}^{\alpha}=-\sum_{j=0}^{k-1} \bar{c}_{j} \cdot P_{\alpha}\left(z^{j}\right) .
$$

Condition (4.7) means that for each $j \in\{0, \ldots, k-1\}$,

$$
0=P_{\beta}\left(z f_{k-j}\right)-c_{j} \cdot P_{\beta} 1=P_{\beta}\left(z f_{k-j}-c_{j}\right) \quad \text { and } \quad z f_{k-j}-c_{j}=\beta \cdot d_{j}
$$

with $d_{j} \in H^{2}$. Moreover, this equality can be written as

$$
\overline{C_{\beta} f_{k-j}}-c_{j} \bar{\beta}=\bar{\beta} z f_{k-j}-c_{j} \bar{\beta}=d_{j},
$$


which implies that $d_{j}$ is also constant. Thus

$$
f_{l}=\frac{d_{k-l} \cdot \beta+c_{k-l}}{z}, \quad 1 \leqslant l \leqslant k
$$

and

$$
\begin{aligned}
z \varphi_{2} & =\sum_{l=1}^{k} z^{l} \cdot W_{k}^{*} f_{l}=\sum_{l=1}^{k} z^{l} \cdot W_{k}^{*}\left(d_{k-l} \bar{z} \beta+c_{k-l} \bar{z}\right)=\left(W_{k}^{*} \beta\right) \cdot \sum_{l=1}^{k} d_{k-l} z^{l} \cdot \bar{z}^{k}+\sum_{l=1}^{k} c_{k-l} \bar{z}^{k-l} \\
& =\bar{z}^{k-1}\left(W_{k}^{*} \beta\right) \cdot \sum_{l=1}^{k} d_{k-l} z^{l-1}+\sum_{j=0}^{k-1} c_{j} \bar{z}^{j} .
\end{aligned}
$$

By (4.5), (4.8), and the fact that $P_{\alpha}\left(z^{j}\right)=z^{j}-\alpha P\left(\bar{\alpha} z^{j}\right)$ we finally get

$$
\begin{aligned}
\varphi= & \overline{\alpha h_{1}}+\left(W_{k}^{*} \beta\right) \cdot z h_{2}+\bar{\varphi}_{1}+z \varphi_{2} \\
= & \overline{\alpha h_{1}}+\left(W_{k}^{*} \beta\right) \cdot z h_{2}+\sum_{j=0}^{k-1} \bar{c}_{j}\left(\alpha P\left(\bar{\alpha} z^{j}\right)-z^{j}\right)+\bar{z}^{k-1}\left(W_{k}^{*} \beta\right) \cdot \sum_{l=1}^{k} d_{k-l} z^{l-1}+\sum_{j=0}^{k-1} c_{j} \bar{z}^{j} \\
= & \overline{\alpha\left(h_{1}+\sum_{j=0}^{k-1} \bar{c}_{j} P\left(\bar{\alpha} z^{j}\right)\right)}-\sum_{j=0}^{k-1} c_{j} \bar{z}^{j}+\bar{z}^{k-1}\left(W_{k}^{*} \beta\right)\left(z^{k} h_{2}+\sum_{l=1}^{k} d_{k-l} z^{l-1}\right)+\sum_{j=0}^{k-1} c_{j} \bar{z}^{j} \\
= & \alpha\left(h_{1}+\sum_{j=0}^{k-1} \bar{c}_{j} P\left(\bar{\alpha} z^{j}\right)\right) \\
&
\end{aligned}
$$

and so $\varphi \in \overline{\alpha H^{2}}+\bar{z}^{k-1}\left(W_{k}^{*} \beta\right) H^{2}$.

Remark.

(a) Note that if $\beta(0)=0$, then

$$
\bar{z}^{k-1}\left(W_{k}^{*} \beta\right) H^{2}=W_{k}^{*}\left(\frac{\beta}{z}\right) z H^{2} \subset z H^{2} .
$$

On the other hand, if $\beta(0) \neq 0$, then

$$
\bar{z}^{k-1}\left(W_{k}^{*} \beta\right) H^{2}=\bar{z}^{k-1}\left(W_{k}^{*} \beta\right)\left(K_{z^{k}} \oplus z^{k} H^{2}\right)=\left(\left(W_{k}^{*} \beta\right) \overline{K_{z^{k}}}\right) \oplus\left(\left(W_{k}^{*} \beta\right) z H^{2}\right) .
$$

(b) For $k=1$, we obtain that $U_{\varphi}^{\alpha, \beta}=A_{\varphi}^{\alpha, \beta}=0$ if and only if

$$
\varphi \in \overline{\alpha H^{2}}+\bar{z}^{k-1}\left(W_{k}^{*} \beta\right) H^{2}=\overline{\alpha H^{2}}+\beta H^{2},
$$

which is the known result for asymmetric truncated Toeplitz operators [22].

Corollary 12. Let $\alpha, \beta$ be two nonconstant inner functions, let $k \in \mathbb{N}$, and assume that $\operatorname{dim} K_{\alpha} \geqslant k, \beta(0)=0$. If $\varphi \in z H^{2}$, then $U_{\varphi}^{\alpha, \beta}=0$ if and only if

$$
\varphi \in \bar{z}^{k-1}\left(W_{k}^{*} \beta\right) H^{2}=W_{k}^{*}\left(\frac{\beta}{z}\right) z H^{2} .
$$


Open Access. This article is licensed under a Creative Commons Attribution 4.0 International License, which permits use, sharing, adaptation, distribution and reproduction in any medium or format, as long as you give appropriate credit to the original author(s) and the source, provide a link to the Creative Commons licence, and indicate if changes were made. The images or other third party material in this article are included in the article's Creative Commons licence, unless indicated otherwise in a credit line to the material. If material is not included in the article's Creative Commons licence and your intended use is not permitted by statutory regulation or exceeds the permitted use, you will need to obtain permission directly from the copyright holder. To view a copy of this licence, visit http://creativecommons.org/licenses/by/4 .0 /

\section{References}

1. S.C. Arora and R. Batra, On generalized slant Toeplitz operators, Indian J. Math., 45(2):121-134, 2003.

2. S.C. Arora and R. Batra, Generalized slant Toeplitz operators on $H^{2}$, Math. Nachr., 278(4):347-355, 2005.

3. A. Böttcher and B. Silbermann, Analysis of Toeplitz Operators, 2nd ed., Springer Monogr. Math., Springer, Berlin, 2006. Prepared jointly with A. Karlovich.

4. C. Câmara, J. Jurasik, K. Kliś-Garlicka, and M. Ptak, Characterizations of asymmetric truncated Toeplitz operators, Banach J. Math. Anal., 11(4):899-922, 2017.

5. M.C. Câmara, K. Kliś-Garlicka, and M. Ptak, Asymmetric truncated Toeplitz operators and conjugations, Filomat, 33(12):3697-3710, 2019.

6. M.C. Câmara and J.R. Partington, Spectral properties of truncated Toeplitz operators by equivalence after extension, J. Math. Anal. Appl., 433(2):762-784, 2016.

7. M.C. Câmara and J.R. Partington, Asymmetric truncated Toeplitz operators and Toeplitz operators with matrix symbol, J. Oper. Theory, 77(2):455-479, 2017.

8. I. Chalendar, E. Fricain, and D. Timotin, A survey of some recent results on truncated Toeplitz operators, in Recent Progress on Operator Theory and Approximation in Spaces of Analytic Functions, Contemp. Math., Vol. 679, AMS, Providence, RI, 2016, pp. 59-77.

9. G. Datt and S.K. Pandey, Compression of slant Toeplitz operators on the Hardy space of $n$-dimensional torus, Czech. Math. J., 70(145)(4):997-1018, 2020.

10. G. Datt and S.K. Pandey, Slant Toeplitz operators on the Lebesgue space of $n$-dimensional torus, Hokkaido Math. J., 49(3):363-389, 2020.

11. P.L. Duren, Theory of $H^{p}$ Spaces, Pure Appl. Math., Vol. 38, Academic Press, New York, London, 1970.

12. E. Fricain and J. Mashreghi, The Theory of $\mathcal{H}(b)$ Spaces, Vol. 1, New Math. Monogr., Vol. 20, Cambridge Univ. Press, Cambridge, 2016.

13. S.R. Garcia, J. Mashreghi, and W.T. Ross, Introduction to Model Spaces and Their Operators, Camb. Stud. Adv. Math., Vol. 148, Cambridge Univ. Press, Cambridge, 2016.

14. S.R. Garcia and M. Putinar, Complex symmetric operators and applications, Trans. Am. Math. Soc., 358(3):12851315, 2006.

15. S.R. Garcia and W.T. Ross, Recent progress on truncated Toeplitz operators, in Blaschke Products and Their Applications, Fields Inst. Commun., Vol. 65, Springer, New York, 2013, pp. 275-319.

16. T.N.T. Goodman, C.A. Micchelli, and J.D. Ward, Spectral radius formulas for subdivision operators, in Recent Advances in Wavelet Analysis, Wavelet Anal. Appl., Vol. 3, Academic Press, Boston, MA, 1994, pp. 335-360.

17. C. Gu, B. Łanucha, and M. Michalska, Characterizations of asymmetric truncated Toeplitz and Hankel operators, Complex Anal. Oper. Theory, 13(3):673-684, 2019. 
18. M.C. Ho, Properties of slant Toeplitz operators, Indiana Univ. Math. J., 45(3):843-862, 1996.

19. M.C. Ho, Adjoints of slant Toeplitz operators, Integral Equations Oper. Theory, 29(3):301-312, 1997.

20. M.C. Ho, Spectra of slant Toeplitz operators with continuous symbols, Mich. Math. J., 44(1):157-166, 1997.

21. M.C. Ho, Adjoints of slant Toeplitz operators. II, Integral Equations Oper. Theory, 41(2):179-188, 2001.

22. J. Jurasik and B. Łanucha, Asymmetric truncated Toeplitz operators equal to the zero operator, Ann. Univ. Mariae Curie-Skłodowska, Sect. A, 70(2):51-62, 2016.

23. J. Jurasik and B. Łanucha, Asymmetric truncated Toeplitz operators on finite-dimensional spaces, Oper. Matrices, 11(1):245-262, 2017.

24. B. Łanucha, On rank-one asymmetric truncated Toeplitz operators on finite-dimensional model spaces, J. Math. Anal. Appl., 454(2):961-980, 2017.

25. B. Łanucha, Asymmetric truncated Toeplitz operators of rank one, Comput. Methods Funct. Theory, 18(2):259-267, 2018.

26. C. Liu and Y. Lu, Product and commutativity of $k$ th-order slant Toeplitz operators, Abstr. Appl. Anal., 2013:473916, 2013.

27. Y. Lu, C. Liu, and J. Yang, Commutativity of $k$ th-order slant Toeplitz operators, Math. Nachr., 283(9):1304-1313, 2010.

28. R.A. Martínez-Avendaño and P. Rosenthal, An Introduction to Operators on the Hardy-Hilbert Space, Grad. Texts Math., Vol. 237, Springer, New York, 2007.

29. D. Sarason, Algebraic properties of truncated Toeplitz operators, Oper. Matrices, 1(4):491-526, 2007.

30. B. Sz.-Nagy, C. Foias, H. Bercovici, and L. Kérchy, Harmonic Analysis of Operators on Hilbert Space, Universitext, Springer, New York, 2010. Revised and enlarged edition.

31. L.F. Villemoes, Wavelet analysis of refinement equations, SIAM J. Math. Anal., 25(5):1433-1460, 1994.

32. T. Zegeye and S. C. Arora, The compression of slant Toeplitz operator to $H^{2}(\partial D)$, Indian J. Pure Appl. Math., 32(2): 221-226, 2001. 